\title{
Time-dependent search of neutrino emission from 2 X-ray and gamma-ray binaries with the ANTARES telescope
}

\author{
Agustín Sánchez Losa* \\ INFN - Sezione di Bari \\ E-mail: agustin.sanchez@ba.infn.it

\section{Damien Dornic} \\ $C P P M$ \\ E-mail: dornic@cppm.in2p3.fr
}

\author{
Alexis Coleiro \\ IFIC \\ E-mail: alexis.coleirodific.uv.es
}

\section{on the behalf of the ANTARES Collaboration}

\begin{abstract}
ANTARES is currently the largest neutrino telescope operating in the Northern Hemisphere, aiming at the detection of high-energy neutrinos from astrophysical sources. Such observations would provide important clues about the processes at work in those objects, and possibly help to understand the origin of very high-energy cosmic rays. By design, neutrino telescopes constantly monitor at least one complete hemisphere of the sky and are thus well set to detect neutrinos produced in transient astrophysical events. The flux of high-energy neutrinos from a transient source is lower than if is an steady one, but the background originating from interactions of charged cosmic rays in the Earth's atmosphere can be drastically reduced by requiring a directional and temporal coincidence of the astrophysical phenomenon detected by electromagnetic instruments. Time-dependent point-source searches have been applied to a list of X-ray and gamma-ray binary systems detected by satellites or TeV imaging Cherenkov telescopes using ANTARES data. The results of these searches are presented. Upper limits on neutrino fluxes, their comparisons with the published gamma-ray spectral energy distribution and with prediction from astrophysical models are also reported.
\end{abstract}

35th International Cosmic Ray Conference - ICRC2017

10-20 July, 2017

Bexco, Busan, Korea

\footnotetext{
*Speaker.
} 


\section{Introduction}

X-ray and gamma-ray binaries (XRBs and $\gamma \mathrm{RBs}$ ) are binary star systems composed of a compact object (e.g. neutron star or stellar mass black hole candidate) orbiting a companion nondegenerate star and that are luminous in X-rays and gamma-rays respectively. The high-energy photon emission of XRBs is due to the matter falling from the companion start into the compact object. On the other hand, in $\gamma \mathrm{RB}$ systems the responsible of the high-energy emission is the interaction of the pulsar wind with the intense stellar wind of the companion massive star. Despite the non-thermal emission is probably dominated by leptonic processes, a hadronic component could also be present. High-energy neutrino emission detection would confirm this possibility and provide insights about the involved acceleration mechanisms that would confirm cosmic ray production on these sources [1]. In a hadronic scenario, the decay of the charged pions produce a neutrino emission correlated with the very high-energy gamma rays from $\pi^{0}$ decays when $\gamma \gamma$ annihilation is negligible. Up to know, a hadronic component has been identified in only two cases [2,3]. Several estimations of the neutrino flux production in these sources are proposed, with very different spectral indexes, cutoffs and normalisations $[4,5,6]$. In order to cover the variety of models accessible to the ANTARES sensitivity, the following neutrino spectra have been considered: $E^{-2}$, $E^{-2} \times \exp (\sqrt{-E / 100 \mathrm{TeV}})$ and $E^{-2} \times \exp (\sqrt{-E / 10 \mathrm{TeV}})$, with $E$ the neutrino energy.

In this contribution is presented a time-dependent analysis realised on the ANTARES [7, 8] neutrino telescope data testing the above hypothesis. This analysis method reduces in a factor of $2-3$ the signal required for a discovery with respect to a time integrated search $[9,10]$ under the assumption of correlation of the neutrino signal with high-energy electromagnetic emission, as is carried out in previous similar analyses [11, 12]. The hadronic hypothesis is tested by looking for a correlation between the neutrino emission and the observed X-ray and gamma-ray flares of the brightest variable XRBs and $\gamma$ RBs (see Sec. 3). The main update with respect to previous analyses is the inclusion of the shower channel in addition to the track one from the ANTARES data taken from 2008 to 2016 with $\sim 2413$ days of live-time.

\section{Time-dependent analysis}

The analysis is done evaluating a test statistic built from a maximised unbinned extended likelihood ratio. The likelihood $(\mathscr{L})$ treats the ANTARES data as a composition of background $\left(\mathscr{N}_{b k}\right)$ and signal $\left(\mathscr{N}_{s g}\right)$, properly weighted by their different probability density functions (PDFs, $\left.P_{s g / b k}\right)$ :

$$
\ln \mathscr{L}_{s g+b k}=\sum_{c h} \sum_{i} \ln \left[\mathscr{N}_{s g}^{c h} \cdot P_{s g}^{c h}+\mathscr{N}_{b k}^{c h} \cdot P_{b k}^{c h}\right]-\left[\mathscr{N}_{s g}+\mathscr{N}_{b k}\right]
$$

where the likelihood is extended over all the different events $(i)$ of each considered channel $(c h)$, i.e. tracks and showers.

The $P_{s g}$ for the track channel is defined as the product of the direction (the point spread function probability, $P S F_{s g}^{t r}(\alpha)$, with $\alpha$ the angular distance to the source), the energy $\left(P_{s g}^{t r}(d E / d X)\right.$, being $d E / d X$ the energy estimator used in the track channel) and the time $\left(P_{s g}(t)\right)$ probabilities:

$$
P_{s g}^{t r}=P S F_{s g}^{t r}\left(\alpha, \delta_{S}\right) \cdot P_{s g}^{t r}(d E / d X) \cdot P_{s g}(t+l a g)
$$


where the PSF is estimated for each source declination $\left(\delta_{S}\right)$ and both $P S F_{s g}^{t r}(\alpha)$ and $P_{s g}^{t r}(d E / d X)$ are dependent of the evaluated spectrum. Additionally, a lag of \pm 5 days is allowed on the neutrino signal arrival time $t$ in order to allow possible offsets between the neutrino and X-ray/gamma-ray emission at leaving the source.

The time probability is the assumed correlation between X-rays/gamma-rays and neutrinos, i.e. the detected signal neutrino time probability is proportional to the X-ray/gamma-ray detection time, PDF extracted from the X-ray/gamma-ray emission of the studied source (see Sec. 3). This time PDF is the same for both track and shower channels.

The term $P_{s g}$ for the shower channel is the product of the shower PSF, the energy and the time PDF:

$$
P_{s g}^{s h}=P S F_{s g}^{s h}\left(\alpha, \delta_{S}\right) \cdot P_{s g}^{s h}\left(n_{h i t s}\right) \cdot P_{s g}(t+l a g)
$$

where the number of hits used in the shower reconstruction, $n_{\text {hits }}$, is used as the energy estimator and again both $P S F_{s g}^{s h}(\alpha)$ and $P_{s g}^{s h}\left(n_{\text {hits }}\right)$ are signal spectrum dependent.

The $P_{b k}$ for each channel are the corresponding products of the background PDF at a certain declination $\left(P_{b k}^{t r}(\delta)\right)$, the background energy estimator PDF and the background time PDF (build from a loser cut on the data sample):

$$
\begin{gathered}
P_{b k}^{t r}=P_{b k}^{t r}(\delta) \cdot P_{b k}^{t r}(d E / d X, \delta) \cdot P_{b k}(t) \\
P_{s g}^{s h}=P_{b k}^{s h}(\delta) \cdot P_{b k}^{s h}\left(n_{h i t s}\right) \cdot P_{b k}(t)
\end{gathered}
$$

where the dependence of the background $d E / d X$ with respect to the declination has been considered.

The amount of signal of each channel is determined by the ratio contribution of each channel to the global acceptance of the detector at source declination:

$$
\mathscr{N}_{s g}^{c h}=\mathscr{N}_{s g} \cdot\left(A_{c c}^{c h}\left(\delta_{S}\right) / A_{c c}^{T O T A L}\left(\delta_{S}\right)\right)
$$

and the total signal or background is the sum of each channel:

$$
\mathscr{N}_{s g / b k}=\mathscr{N}_{s g / b k}^{s h}+\mathscr{N}_{s g / b k}^{t r}
$$

The likelihood is maximised by varying the $\mathscr{N}_{s g}$ and lag parameters and the test statistic $\mathscr{Q}$ is built from the ratio of this maximised likelihood with the likelihood value corresponding to the null hypothesis:

$$
\mathscr{Q}=\log \mathscr{L}_{s g+b k}^{\max }-\log \mathscr{L}_{b k}
$$

The significance of this test statistic is evaluated via pseudo-experiments. Track quality cuts are optimized on a source and spectrum basis in order to maximize the model discovery potential at $3 \sigma$. For the shower channels, the quality cuts optimized for the latest point source analysis are considered.

\section{Source and flare selection}

Under the assumption of correlated high-energy neutrino and electromagnetic productions, $\mathrm{X}$-ray and gamma-ray variable emissions from XRBs and $\gamma \mathrm{RBs}$ are used to build the neutrino emission time PDFs. The approach for each kind of source differs due to their emissions at different energies. 


\subsection{X-ray binary source and flare selection}

XRBs exhibiting outburst periods are selected from the Swift [13] and MAXI [14] catalogues, extended with Rossi [15] data when available. XRB light curves (LCs) are obtained from:

- Swift/BAT Hard X-ray Transient Monitor ${ }^{1}$ : any high-mass XRB (HMXR) and low-mass XRB (LMXB) with significant time variabilities are initially selected. Their daily LCs are denoised with a maximum likelihood block [16] procedure and their flare significance characterised as done in previous analyses $[12,17]$. Sources with more than one flare above a 5 standard deviation significance are selected for the analysis.

- MAXI Light Curves ${ }^{2}$ : The same procedure as for Swift LCs is followed for select MAXI galactic compact binary flares.

- RXTE/ASM Light Curves ${ }^{3}$ : Because X-ray data are not always available for all the sources from the above detectors, in order to cover possible flares previously to 2012, Rossi LCs have been also considered in the same way as the other telescopes.

Depending on the time period and the availability of the different instruments, outbursts are better observed in one apparatus compared to others. Therefore, Swift flare selection is completed with the flares only observed in the other telescopes. The merging of the different LCs is done by normalising each detector $\mathrm{LC}$ to its relative significance.

The final source list comprises 36 XRBs (see Table 1), including 14 HMXBs and 19 LMXBs, half in common with the previous analysis [12] since faint sources are removed and XRBs flaring in 2014-2016 added.

\subsection{Gamma-ray binary source and flare selection}

Four $\gamma$ RBs compatible with ANTARES up-going visibility have been selected for the study at very high-energy gamma-rays: 1FGL J1018.6-5856 [18], HESS J0632+057 [19], LS 5039-63 [20] and PSR B1259-63 [21]. Using their periodic emission established in the literature, simple on/off LCs (considering the parameter uncertainties in the flare period definition) are used for their time PDFs (see Table 2), using for LS 5039 its TeV flaring information and not the $\mathrm{GeV}$ one.

Additionally, the Cyg X-3 XRB has been detected outbursting at gamma-ray energies [22] by the Fermi-LAT telescope [23]. Thus, Cyg $\mathrm{X}-3$ is included in the analysis using the multiwavelenght flare observations published in [22] (Table 2, Y+ and $\mathrm{Y}-$ criteria) and updated with two astronomic alerts ${ }^{4}$ : 57398-54412MJD (\#ATel 8591) and 57646-57647MJD (\#ATel 9502). The same on/off criteria used for the other $\gamma \mathrm{RB}$ s is applied also here for the LC construction, adding \pm 0.5 day on the begin and end of the flare definition periods.

\footnotetext{
${ }^{1}$ https://swift.gsfc.nasa.gov/results/transients

${ }^{2}$ http://134.160.243.77/top/lc.html

${ }^{3}$ http://xte.mit.edu/ASM_lc.html

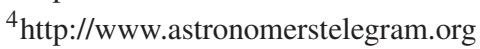


Table 1: List of the 36 XRBs selected for the analysis. For each source the satellite LC used (Swift, MAXI or Rossi), the number of flares (\#flares), the flaring days, the source right ascension and its declination are listed.

\begin{tabular}{lcrr}
\hline Name & Satellite $(\#$ flares $\mid$ days $)$ & R.A. $\left(^{\circ}\right)$ & $\delta\left(^{\circ}\right)$ \\
\hline 1A 0535+262 & $\mathrm{S}(\# 11 \mid 417)+\mathrm{M}(\# 2 \mid 30)$ & 84.7 & 26.3 \\
1A 1118-61 & $\mathrm{S}(\# 1 \mid 141)$ & 170.2 & -61.9 \\
1A 1742-294 & $\mathrm{S}(\# 1 \mid 3)+\mathrm{M}(\# 5 \mid 284)$ & 266.5 & -29.5 \\
4U 1630-472 & $\mathrm{S}(\# 6 \mid 437)+\mathrm{M}(\# 3 \mid 278)$ & 248.5 & -47.4 \\
Aql X-1 & $\mathrm{S}(\# 7 \mid 460)+\mathrm{M}(\# 10 \mid 95)$ & 287.8 & 0.6 \\
AX J1749.1-2639 & $\mathrm{S}(\# 1 \mid 85)$ & 267.3 & -26.6 \\
Cir X-1 & $\mathrm{S}(\# 10 \mid 205)+\mathrm{M}(\# 18 \mid 478)$ & 230.2 & -57.2 \\
Cyg X-1 & $\mathrm{S}(\# 9 \mid 1965)$ & 299.6 & 35.2 \\
EXO 1745-248 & $\mathrm{S}(\# 3 \mid 191)+\mathrm{M}(\# 4 \mid 237)$ & 267.0 & -24.8 \\
GRO J1008-57 & $\mathrm{S}(\# 12 \mid 614)$ & 152.4 & -58.3 \\
GRS 1739-278 & $\mathrm{S}(\# 1 \mid 143)+\mathrm{M}(\# 2 \mid 264)$ & 265.7 & -27.8 \\
GS 0834-430 & $\mathrm{S}(\# 1 \mid 1427)+\mathrm{M}(\# 2 \mid 13)$ & 129.0 & -43.2 \\
GS 1354-64 & $\mathrm{S}(\# 1 \mid 136)+\mathrm{M}(\# 3 \mid 16)$ & 209.5 & -64.7 \\
GX 1+4 & $\mathrm{S}(\# 9 \mid 661)+\mathrm{M}(\# 2 \mid 58)+\mathrm{R}(\# 1 \mid 93)$ & 263.0 & -24.7 \\
GX 304-1 & $\mathrm{S}(\# 16 \mid 579)+\mathrm{M}(\# 1 \mid 10)$ & 195.3 & -61.6 \\
GX 339-4 & $\mathrm{S}(\# 5 \mid 525)+\mathrm{M}(\# 5 \mid 121)$ & 255.7 & -48.8 \\
H 1417-624 & $\mathrm{S}(\# 1 \mid 107)$ & 215.3 & -62.7 \\
H 1608-522 & $\mathrm{S}(\# 7 \mid 967)+\mathrm{M}(\# 12 \mid 384)$ & 243.2 & -52.4 \\
H 1743-322 & $\mathrm{S}(\# 12 \mid 772)+\mathrm{M}(\# 3 \mid 33)$ & 266.6 & -32.2 \\
IGR J17473-2721 & $\mathrm{S}(\# 1 \mid 9)+\mathrm{R}(\# 1 \mid 61)$ & 266.8 & -27.3 \\
KS 1947+300 & $\mathrm{S}(\# 4 \mid 324)+\mathrm{M}(\# 10 \mid 242)$ & 297.4 & 30.2 \\
MAXI J0556-332 & $\mathrm{M}(\# 2 \mid 475)$ & 89.2 & -33.2 \\
MAXI J1543-564 & $\mathrm{M}(\# 3 \mid 131)$ & 235.8 & -56.4 \\
MAXI J1659-152 & $\mathrm{S}(\# 2 \mid 125)+\mathrm{R}(\# 2 \mid 96)$ & 254.8 & -15.3 \\
MAXI J1836-194 & $\mathrm{S}(\# 1 \mid 83)+\mathrm{M}(\# 2 \mid 18)$ & 278.9 & -19.3 \\
MXB 0656-072 & $\mathrm{S}(\# 1 \mid 37)+\mathrm{M}(\# 1 \mid 2)+\mathrm{R}(\# 1 \mid 4)$ & 104.6 & -7.2 \\
SAX J1747.0-2853 & $\mathrm{M}(\# 6 \mid 382)$ & 266.8 & -28.9 \\
SMC X-3 & $\mathrm{S}(\# 1 \mid 90)+\mathrm{M}(\# 1 \mid 3)$ & 13.0 & -72.4 \\
SWIFT J1539.2-6227 & $\mathrm{S}(\# 1 \mid 46)$ & 234.8 & -62.5 \\
SWIFT J1745.1-2624 & $\mathrm{S}(\# 1 \mid 198)$ & 266.3 & -26.4 \\
SWIFT J1842.5-1124 & $\mathrm{S}(\# 1 \mid 133)+\mathrm{R}(\# 1 \mid 356)$ & 280.6 & -11.4 \\
SWIFT J1910.2-0546 & $\mathrm{S}(\# 2 \mid 52)+\mathrm{M}(\# 2 \mid 14)$ & 287.6 & -5.8 \\
V404 Cyg & $\mathrm{S}(\# 2 \mid 210)+\mathrm{M}(\# 12 \mid 229)$ & 268.1 & -22.3 \\
XTE J1752-223 & $\mathrm{M}(\# 1 \mid 12)$ & -19.1 \\
XTE J1810-189 & 296.4 & 27.4 \\
XTE J1946+274 & &
\end{tabular}


Table 2: List of the $4 \gamma$ RBs selected for the analysis. For each source are given its coordinates and are listed the period, flaring phase and periastron extracted from the bibliography and used to build their time PDFs.

\begin{tabular}{lrrccc}
\hline Name & R.A. $\left({ }^{\circ}\right)$ & $\delta\left(^{\circ}\right)$ & Period (days) & Flaring phase & Periastron (MJD) \\
\hline 1FGL J1018.6-5856 & 154.7 & -58.9 & $16.58 \pm 0.02$ & $0.70-0.40$ & $55387.5 \pm 0.4$ \\
HESS J0632+057 & 98.2 & 5.8 & $315 \pm 5$ & $0.20-0.45$ & $54587.0 \pm 0.5$ \\
LS 5039-63 & 276.6 & -14.8 & $3.91 \pm 8 \cdot 10^{-5}$ & $0.45-0.95$ & $51942.59 \pm 0.05$ \\
PSR B1259-63 & 195.7 & -63.8 & $1236.7 \pm 2 \cdot 10^{-5}$ & $0.92-0.08$ & $55545.0 \pm 0.5$ \\
\hline
\end{tabular}

\section{Results}

Preliminary sensitivities for the track only channel considering a $E^{-2}$ spectrum have been presented at the conference. Some specific cases are presented in Tables 3 and 4 for both XRBs and $\gamma \mathrm{RBs}$ respectively. Sensitivity to the neutrino flux during the flares, source dependent, is improved by a factor of $\sim 4$ on average with respect to the previous analysis upper limits. In function of the source declination, shower channel inclusion would improve neutrino limits even a $10 \%$. The corresponding neutrino fluence estimate is also provided in the tables according to its definition:

$$
\mathscr{F}=\iint E \frac{\mathrm{d} N}{\mathrm{~d} E} \mathrm{~d} E \mathrm{~d} t=\phi_{0} \Delta t \int_{5 \%}^{95 \%} E E^{-2} \mathrm{~d} E
$$

with $\phi_{0}$ the spectrum normalisation, $\Delta t$ the flaring livetime and the integral performed in the 5-95\% ANTARES sensibility energy range of each source. The whole sample is expected to be unblinded in the near future.

Table 3: Preliminary sensitivities for some specific XRBs for the track only channel assuming a $E^{-2}$ spectrum. For each source are given the neutrino flux sensitivity during the flare $\left(\phi_{0}\right.$, in $\left.10^{-8} \mathrm{GeV}^{-1} \mathrm{~cm}^{-2} \mathrm{~s}^{-1}\right)$, the flare livetime ( $\Delta t$, in days), the integral in the 5-95\% ANTARES sensibility energy range $\left(I_{5 \%}^{95 \%}=\right.$ $\int_{5 \%}^{95 \%} E^{-1} \mathrm{~d} E$ ) and the fluence $\left(\mathscr{F}\right.$, in $\left.\mathrm{GeV} \mathrm{cm}^{-2}\right)$.

\begin{tabular}{lcccc|lcccc}
\hline Name & $\phi_{0}$ & $\Delta t$ & $I_{5 \%}^{95 \%}$ & $\mathscr{F}$ & Name & $\phi_{0}$ & $\Delta t$ & $I_{5 \%}^{95 \%}$ & $\mathscr{F}$ \\
\hline 1A 0535+262 & 8.6 & 278 & 7.0 & 14 & GX 339-4 & 2.5 & 464 & 7.2 & 7.1 \\
4U 1630-472 & 2.0 & 579 & 7.2 & 7.1 & H 1743-322 & 2.1 & 665 & 7.4 & 8.9 \\
Cir X-1 & 2.0 & 572 & 6.8 & 6.8 & SMC X-3 & 13 & 88 & 6.5 & 6.3 \\
Cyg X-1 & 1.8 & 1521 & 7.1 & 17 & V404 Cyg & 22 & 120 & 7.1 & 17 \\
\hline
\end{tabular}

Table 4: Preliminary sensitivities for the studied $\gamma \mathrm{RBs}$ for the track only channel assuming a $E^{-2}$ spectrum. For each source are given the neutrino flux sensitivity during the flare $\left(\phi_{0}\right.$, in $\left.10^{-8} \mathrm{GeV}^{-1} \mathrm{~cm}^{-2} \mathrm{~s}^{-1}\right)$, the flare livetime ( $\Delta t$, in days), the integral in the 5-95\% ANTARES sensibility energy range $\left(I_{5 \%}^{95 \%}=\int_{5 \%}^{95 \%} E^{-1} \mathrm{~d} E\right)$ and the fluence $\left(\mathscr{F}\right.$, in $\left.\mathrm{GeV} \mathrm{cm}^{-2}\right)$.

\begin{tabular}{lcccc|lcccc}
\hline Name & $\phi_{0}$ & $\Delta t$ & $I_{5 \%}^{95 \%}$ & $\mathscr{F}$ & Name & $\phi_{0}$ & $\Delta t$ & $I_{5 \%}^{95 \%}$ & $\mathscr{F}$ \\
\hline 1FGL J1018.6-5856 & 0.5 & 2259 & 6.7 & 6.8 & LS 5039-63 & 1.1 & 1564 & 7.1 & 10 \\
Cyg X-3 & 146 & 20 & 7.2 & 18 & PSR B1259-63 & 3.0 & 377 & 6.6 & 6.5 \\
HESS J0632+057 & 1.6 & 1219 & 7.0 & 12 & & & & & \\
\hline
\end{tabular}




\section{References}

[1] J.K. Becker, High-energy neutrinos in the context of multimessenger physics, Phys. Rep. 548 (2008) 173 [astro-ph/0710.1557].

[2] S. Migliari, R. Fender, M. Mendez, Iron emission lines from extended x-ray jets in SS 433: reheating of atomic nuclei, Science 297 (2002) 1673.

[3] M.D. Trigo, J.C.A. Miller-Jones, S. Migliari, J.W. Broderick, T. Tzioumis, Baryons in the Relativistic Jets of the Stellar-Mass Black-Hole Candidate 4U 1630-47, Nature 504 (2013) 260 .

[4] C. Distefano, D. Guetta, E. Waxman, A. Levinson, ApJ 575 (2002) 378 .

[5] G.E. Romero, D.F. Torres, M.M. Kaufman Bernadò, I.F. Mirabel, A\&A 410 (2003) L1 .

[6] A. Levinson, E. Waxman, Physical Review Letters 87 (2001) 171101.

[7] J.A. Aguilar et al. (the ANTARES Collaboration), ANTARES: the first undersea neutrino telescope, Nuclear Inst. and Methods in Physics Research A 656 (2011) 11 [astro-ph/1104 .1607].

[8] A. Heijboer, Highlights from the ANTARES neutrino telescope, in proceedings of ICRC2017, PoS ( ICRC2017) 002 (2017).

[9] A. Albert et al. (the ANTARES Collaboration), First all-flavour Neutrino Point-like Source Search with the ANTARES Neutrino Telescope, astro-ph/1706.01857.

[10] G. Illuminati, All-flavor Neutrino Point-like Source Search with the ANTARES Neutrino Telescope, in proceedings of ICRC2017, POS ( ICRC2017) NU055 (2017).

[11] S. Adrián-Martínez et al. (the ANTARES Collaboration), A search for time dependent neutrino emission from microquasars with the ANTARES telescope, JHEAp 3-4 (2014) 9 [astro-ph/1402.1600].

[12] A. Albert et al. (the ANTARES Collaboration), Time-dependent search for neutrino emission from X-ray binaries with the ANTARES telescope, JCAP 04 (2017) 019 [astro-ph/1609. 07372] .

[13] The Swift homepage: http://swift.gsfc.nasa.gov/

[14] The MAXI homepage: http://maxi.riken.jp

[15] The Rossi homepage: http://heasarc.gsfc.nasa.gov/docs/xte/XTE.html

[16] J.D. Scargle, J.P. Norris, B. Jackson, J. Chiang, Studies in Astronomical Time Series Analysis. VI. Bayesian Block Representations, The Astrophysical Journal 764 (2013) 167 [astro-ph/1207.5578].

[17] S. Adrián-Martínez et al. (the ANTARES Collaboration), Search for muon-neutrino emission from GeV and TeV gamma-ray flaring blazars using five years of data of the ANTARES telescope, JCAP 12 (2015) 014 [astro-ph/1506.07354].

[18] M.J. Coe, F. Di Mille, P.G. Edwards, M.D. Filipović, J.L. Payne, J. Stevens, M.A.P. Torres (the Fermi LAT Collaboration), Periodic Emission from the Gamma-ray Binary 1FGL J1018.6-5856, Science 335 (2012) 6065 [astro-ph/1202.3164].

[19] S. Bongiorno, A. Falcone, M. Stroh, J. Holder, J. Skilton, J. Hinton, N. Gehrels, J. Grube, A New TeV Binary: The Discovery of an Orbital Period in HESS J0632+057, The Astrophysical Journal Letters 737 (2011) 1 [astro-ph/1104.4519].

[20] J. Casares, M. Ribo, I. Ribas, J.M. Paredes, J. Marti, A. Herrero, A possible black hole in the gamma-ray microquasar LS 5039, Mon Not R Astron Soc 364 (2005) 3 [astro-ph/ 0507549 ] . 
[21] A. Abramowski et al. (the H.E.S.S. Collaboration), H.E.S.S. Observations of the Binary System PSR B1259-63/LS 2883 around the 2010/2011 Periastron Passage, A\&A 551 (2013) A94 [astro-ph/1301.3930].

[22] A. Bodaghee, J.A. Tomsick, K. Pottschmidt, J. Rodriguez, J. Wilms, G.G. Pooley, Gamma-ray observations of the microquasars Cygnus X-1, Cygnus X-3, GRS 1915+105, and GX 339-4 with the Fermi Large Area Telescope, The Astrophysical Journal 775 (2013) 2 [astro-ph/1307 . 32 64]

[23] The Fermi LAT homepage: https://www-glast.stanford.edu/ 